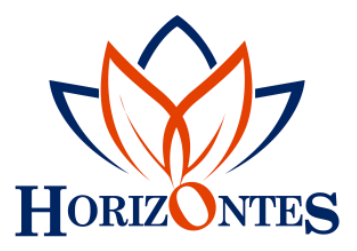

www.revistahorizontes.org
Horizontes. Revista de Investigación en Ciencias de la Educación Https://doi.org/10.33996/revistahorizontes.v5i17.166

Enero - marzo 2021

Volumen 5 / No. 17

ISSN: $2616-7964$

ISSN-L: $2616-7964$

pp. $178-194$

\title{
Liderazgo pedagógico y el desempeño docente: la imagen de instituciones educativas privadas
}

Pedagogical leadership and teaching performance: the image from private educational institutions

Liderança pedagógica e atuação docente: a imagem de instituições educacionais privadas

\section{ARTÍCULO DE INVESTIGACIÓN}

\section{Salomón Vásquez Villanueva}

salomonv@upeu.edu.pe

ORCID: 0000-0001-8824-6176

Universidad Peruana Unión, Lima, Perú

Lizardo Vásquez Villanueva

lizar_v@upeu.edu.pe

ORCID: 0000-0002-1516-1312
Salomón Axel Vásquez Campos

salomonvasquez@upeu.edu.pe

ORCID: 0000-0001-9405-0794

Universidad Peruana Unión, Lima, Perú

Michel Francisco Carranza Quevedo

michelcq25@hotmail.com

ORCID: 0000-0003-0244-5727

Universidad Adventista de Chile, Chile

\author{
Otto Franklin Terry-Ponte \\ oterry@ucv.edu.pe \\ ORCID: 0000-0003-0717-8023
}

Universidad César Vallejo, Perú

Universidad Peruana Unión, Lima, Perú Universidad César Vallejo, Perú

Recibido 13 de febrero 2021 | Arbitrado y aceptado 02 de marzo 2021 | Publicado en marzo 2021

\section{RESUMEN}

La investigación fue diseñada para lograr el objetivo: determinar la relación entre el liderazgo pedagógico y el desempeño docente, realizada en la Asociación Educativa Adventista Nor Oriental, del Perú, en 75 participantes, quienes contestaron los dos instrumentos, para medir las dos variables, en un diseño descriptivo y correlacional, con enfoque cuantitativo. El análisis estadístico de correlación de Rho Spearman presenta el resultado de 0,543 y un $\mathrm{p}$ valor igual a 0.000 (p valor $<0,05$ ); en conclusión, el liderazgo pedagógico se relaciona significativamente con el desempeño docente; dicho de otro modo, la relación entre las variables estudiadas es directa; es decir, a mejor liderazgo pedagógico, mejor será el desempeño docente.

Palabras clave: Liderazgo pedagógico; liderazgo participativo; liderazgo consultivo; desempeño docente

\section{ABSTRACT}

The research was designed to achieve the objective: to determine the relationship between pedagogical leadership and teaching performance, carried out in the North Eastern Adventist Educational Association, of Peru, in 75 participants, who answered the two instruments, to measure the two variables, in a descriptive and correlational design, with a quantitative approach. The Rho Spearman statistical correlation analysis presents the result of 0.543 and a $p$ value equal to 0.000 ( $p$ value $<0.05$ ); In conclusion, pedagogical leadership is significantly related to teaching performance; In other words, the relationship between the variables studied is direct; in other words, the better pedagogical leadership, the better the teaching performance.

Key words: Pedagogical leadership; participatory leadership; consultative leadership; teaching performance 


\section{RESUMO}

A pesquisa foi elaborada para atingir o objetivo: determinar a relação entre liderança pedagógica e desempenho docente, realizada na Associação Educacional Adventista do Nordeste, do Peru, em 75 participantes, que responderam aos dois instrumentos, para mensurar as duas variáveis, em um desenho descritivo e correlacional, com abordagem quantitativa. A análise estatística de correlação de Rho Spearman apresenta o resultado de 0,543 e um valor de p igual a 0,000 (valor de $p<0,05$ ); em conclusão, a liderança pedagógica está significativamente relacionada ao desempenho docente; em outras palavras, a relação entre as variáveis estudadas é direta; em outras palavras, quanto melhor liderança pedagógica, melhor o desempenho docente.

Palavras-chave: Liderança pedagógica; liderança participativa; liderança consultiva; desempenho docente

\section{INTRODUCCIÓN}

El estudio abordó el liderazgo pedagógico y el desempeño docente, con la inquietud de analizar el comportamiento de las dos variables, en las instituciones educativas particulares, en una región del Perú. Las instituciones educativas demandan las capacidades y competencias del liderazgo pedagógico (Roca y Alonso, 2020), también las competencias directivas (Vela, Cáceres, Vela y Gamero, 2020), el desarrollo de la dimensión afectiva (Gento, González y Silfa, 2020). En la Pontificia Universidad Católica de Valparaíso Chile, Quiroga y Aravena (2017) realizan su estudio cualitativo sobre el liderazgo pedagógico en noveles de las Unidades Técnico Pedagógicas, apelando a un carácter descriptivo, realizado con la participación de cinco jefas novelas. En el estudio se subraya, a modo de conclusión, el desafío personal y la disposición para el aprendizaje, el análisis sistemático (demandas y requerimientos), la transición de la docencia a la jefatura.

Llorent, Cobano y Navarro (2017) realizan su investigación con el objetivo de "coadyuvar a la toma de conciencia sobre la necesidad de que los directores puedan ejercer un liderazgo pedagógico, especialmente en escuelas situadas en contextos desfavorecidos" (p. 546), en una población de 955 docentes, cuya muestra fue 280 , determinada mediante un "muestreo estratificado proporcional", además fueron entrevistados 14 directores. Los datos fueron recabados mediante dos instrumentos: el cuestionario y la entrevista semiestructurada, logrando de esta manera la investigación mixta: cuantitativa y cualitativa. Durante la entrevista se abordaron: "a) supervisión de la enseñanza; b) desarrollo profesional docente; y, c) apertura externa". En el estudio cuantitativo, se utilizó la escala tipo Likert, cuyas opciones fueron cinco (1=nunca; 5=siempre). Entre sus resultados, los directores en su mayoría manifestaron que no realizan supervisión en las aulas, solamente un porcentaje menor sí lo realizaba. También revelaron que no tenían la autoridad pedagógica para supervisar a los docentes, por falta de conocimiento y preparación; además, cada profesor tiene autonomía y libertad de cátedra, por la confianza en los profesores; se percibe una cuestión de violencia, "pudiendo perjudicar su relación con el profesorado". Los profesores en su mayoría conciben que los directores fomentan la formación continua y con bastante frecuencia; sin embrago, el 26,2\% piensa lo contrario. Se realiza una mayor supresión sobre los profesores noveles. Se evidencia la poca potestad de los directores "dificultando así el deseable feedback con el profesorado".

Se percibe "un mayor liderazgo pedagógico minimizando los atisbos individualistas en las prácticas de enseñanza" (p. 560). Llegan a la conclusión de que los directores requieren un "desempeño de una formación continua específica en liderazgo pedagógico, que les capacite para poner en marcha buenas prácticas" (p. 561). Son necesarios e 
imprescindibles: la formación, la capacitación y la evaluación del desempeño docente, el cual ha sido descuidado y desvinculado de los resultados académicos y de los quehaceres en el aula (Ramos \& Rueda, 2020). En el desempeño docente, juegan mucha importancia las expectativas y el compromiso de los docentes, el trabajo en equipo, el desarrollo profesional, el clima escolar, el nivel de la institución educativa (Penichel, Ramón, Guzmán, \& Mora, 2020). El desempeño docente adolece de evaluaciones formativas (Ramos \& Rueda, 2020), ha descuidado el conocimiento y los análisis de los factores que lo afectan (Penichel et al., 2020), tampoco conoce las fortalezas y los aspectos que lo mejoran (Quiriz \& Tobón, 2019), cómo se relaciona con el compromiso organizacional (Estrada \& Mamani, 2020), con la gestión pedagógica (Quispe, 2020). En este contexto, se consideró pertinente atender el objetivo: determinar la relación entre el liderazgo pedagógico y el desempeño docente, procediéndose a desarrollar un corpus teórico que dé claridad y fuerza a los análisis y las interpretaciones de los datos recabados.

\section{Liderazgo pedagógico}

\section{Concepciones diversas sobre el liderazgo pedagógico}

De acuerdo con Arellano y Zapata en Fernández y Martín (2014), el liderazgo transforma la realidad, es efectivo, necesario para la organización, la planificación y la participación, "influye, inspira y moviliza las acciones de la comunidad educativa en función de lo pedagógico" (Jiménez, 2017, p. 23). Para Rodríguez (2011, en Jiménez, 2017),

el liderazgo pedagógico es la capacidad de movilizar e influenciar a otros para lograr aprendizajes en todos sus estudiantes, promoviendo sistemas de acompañamiento y retroalimentación de las prácticas docentes, generan instancias e instrumentos de supervisión en conjunto, que a largo plazo influyen en procesos de evaluación y superación docente (p. 24).

Se ha constituido "en una competencia esencial para mejorar la calidad educativa" (Mellado, Chaucono y Villagra, 2017). Es una disciplina, cuyo líder revela "compromiso consigo mismo" y "ejerce una influencia especial" sobre las personas, quienes son lideradas (Hurtado, 2017).

Para Saavedra (2018),
el liderazgo pedagógico es el proceso por el cual el director influye sobre los aprendizajes de los estudiantes creando entornos de aprendizajes para los maestros, monitoreándolos constantemente, motivándolos y orientándolos sobre estrategias de mejora en su práctica pedagógica, de esta manera contribuirá a mejorar su desempeño y por ende se elevará el rendimiento de los estudiantes (p. 38).

\section{Propósito del liderazgo pedagógico}

Es imposible dejar al margen la preocupación centrada en la educación, en el contexto de Iberoamérica, espacio en el cual quedan adscritos, quienes conducen las consignas de profesores, estudiantes, administradores, gestores y líderes. En este panorama de la educación y el liderazgo pedagógico, ¿cuál es el rol de los profesores?

Todos los análisis sobre la calidad de la educación de Iberoamérica 
coinciden en considerar al profesorado como el factor más importante y decisivo para llevar a cabo políticas eficaces de mejora y, en consecuencia, que es imprescindible ofrecerle el más generoso apoyo, alta consideración, formación inicial y continua, estima, mejores retribuciones y suficientes medios. Es una especie de figura de culto cuya bondad generalizada se presupone, así como que es merecedor de los mayores esfuerzos y reconocimientos por el conjunto de la sociedad (Jabonero Blanco, en Fernández y Martín, 2014, p. 11).

Para Roldán (2014), el liderazgo pedagógico es

el conjunto de acciones que el directivo desarrolla para motivar a toda la comunidad educativa a brindar un servicio educativo de calidad que se refleje en el nivel de aprendizaje optimo que logren sus estudiantes comunicando para ello al docente el gran cambio que debe realizar en su labor y motivando en el estudiante las ganas de aprender (p. 31).

Este liderazgo "se ha convertido en una competencia esencial para mejorar la calidad educativa" (Mellado et al., 2017, p.542). El liderazgo del director (líder principal) es considerado "favorecedor de unos mejores resultados académicos del alumnado, siendo mayor esta influencia $\mathrm{y}$, por tanto, más necesaria, en escuelas situadas en entornos desfavorecidos" (Llorent et al. (2017, p. 546). Por otro lado, es "un importante mecanismo para mejorar el aprendizaje del alumnado que más lo necesita" (Llorent et al., 2017, p. 543). Además "emplaza al directivo a desarrollar capacidades profesionales docentes al interior del centro educativo" (Mellado et al., 2017, p. 542).

El liderazgo pedagógico, sobre la base de una buena gestión curricular, busca fortalecer, mejorar el desempeño del docente, constituyendo comunidades de docentes buscadores de los aprendizajes requeridos, gracias al acompañamiento, el monitoreo y la evaluación pertinente, sobre la formación pedagógica y didáctica, cuyas estrategias respalden los estilos y los ritmos de los aprendizajes. En una frase, busca la "mejora de los aprendizajes” (Fernández, 2018).

Son necesarios los planes de acción, los cuales mejorarán los liderazgos pedagógicos de los docentes, fortaleciendo de esta manera los procesos de enseñanza y aprendizaje, dejando entre los alumnos el desarrollo de las competencias esperadas. Son requeridas las estrategias pedagógicas (Cortez, 2018). Los directores desempeña una función, cuyo rol es el liderazgo pedagógico, quien fortalece las competencias de los docentes, facilitándoles el uso estrategias pedagógicas innovadoras, también crea las condiciones favorables para el trabajo docente orientado a lograr los aprendizajes, mediante los medios, los materiales y los recursos pertinentes (Villegas, 2018).

\section{Dimensiones del liderazgo pedagógico}

¿El liderazgo pedagógico tiene dimensiones, cuáles son? Roldán (2014) aborda las siguientes dimensiones: gestión de las condiciones, orientación de los procesos pedagógicos (preparación para el aprendizaje y enseñanza para el aprendizaje), participación en la gestión, desarrollo profesional y la identidad del docente. En este sentido, los directores hacen el esfuerzo para desarrollar las dimensiones: 
1) Planeamiento estratégico: capacidad de establecer una visión y cursos de acción acordes con las necesidades educativas del contexto, 2) Liderazgo: capacidad de movilizar e influir en otros para desarrollar comprensiones compartidas en un contexto profesional no coercitivo, 3) Comunicación: capacidad de generar relaciones de confianza promoviendo el trabajo colegiado y un clima propicio para el aprendizaje y 4) Gestión curricular: capacidad de priorizar y desarrollar prácticas dirigidas a mejorar los procesos de enseñanza y aprendizaje (Romero, 2016, p. 475)

\section{Teorías del liderazgo pedagógico}

Se ha encontrado la Teoría de los rasgos: personalidad, condiciones intelectuales (Murillo, 2008, en Jiménez, 2017), Teoría de la conducta: conducta relacionada con las funciones (Murillo, 2008, en Jiménez, 2017), Teorías implícitas, llamadas teorías espontáneas que comprende teoría directa, teoría interpretativa y teoría constructiva (Vela, 2017).

\section{Desempeño docente}

\section{El desempeño docente: sus concepciones}

La educación se extiende más allá de las habilidades técnicas, busca la sabiduría y el discernimiento, cultivados en los estudiantes, en la condición de niños y jóvenes, también "el desarrollo personal y moral" (Carr, 2003, en González y Subaldo, 2015). El desempeño docente es uno de los factores principales "de la calidad del sistema educativo" (Flores, 2012, en Flores, Sánchez y Martínez, 2016).

El desempeño docente depende de la formación inicial del docente, que depende de las políticas y de la calidad de la institución superior, la cual persigue el desempeño docente que "demuestre las competencias, los métodos de enseñanza y aprendizaje más eficaces en beneficio de los estudiantes, práctica que se refleja de manera inmediata en el quehacer educativo, dentro de su área de desempeño y en la relación con los estudiantes" (Martínez, Castillo y Granda, 2017, p. 84).

El desempeño docente es concebido en términos de proceso, cuyas características son: formativo, sumativo, constructivo, con la pretensión de logar cambios axiológicos deseables y valiosos, sistemáticos, válidos, fiables, en el contexto del logro de los objetivos propuestos (Ralph, 1999; Valdés, 2002; en Martínez et al., 2017). Es el quehacer del docente antes, durante y después del episodio didáctico, comprende la planeación, la impartición de las clases, la revisión de los trabajos, las tareas básicas y complementarias, también las evaluación de los procesos del aprendizaje (Ramos y Rueda, 2020).

\section{Dimensiones del desempeño docente}

Por su parte, Pimienta (2014) elaboró y validó un instrumento para la medición del desempeño docente, cuyo instrumento comprende las dimensiones: planeación, didáctica, aspectos relacionales, ofreciendo un total de 30 indicadores. En el Perú, el Ministerio de Educación diseñó y publicó el documento denominado "Marco de Buen Desempeño Docente" (2012), en el cual se registran cuatro dominios: "Preparación para el aprendizaje de los estudiantes", "Enseñanza para el aprendizaje de los estudiantes", "Participación en la gestión de la escuela articulada a la comunidad" y "Desarrollo de la profesionalidad y la identidad docente" (González y Subaldo, 2015). 
Entre otras, las dimensiones del desempeño docente a considerar: "dominio disciplinar" (conocimiento, manejo de contenidos, programas, diseño, práctica docente), "aspectos didácticos" (evaluación, simuladores, estudio de casos), "pensamiento didáctico" (cuestionarios, creencias, ideas, presentación de casos), "motivación, autoeficacia y responsabilidad docente": "Cuestionario para maestros, viñetas y casos" (Carlos, 2016).

El docente se enmarca y "desarrolla acciones docentes, de investigación, de difusión $\mathrm{y}$ de servicio, basadas en el cono- cimiento científico, la calidad académica, la capacidad de innovación, la ética y el humanismo" (Flores, Gatica, Sánchez y Martínez, 2017, p. 97).

Se ha trabajado el desempeño docente con enfoque de competencias, en el cual se ha trabajado cinco dimensiones: "Trabajo Docente educativo, Trabajo político-ideológico, Trabajo metodológico, Trabajo de Investigación e innovación y Superación" (Ortiz, Borges, Rodríguez, Sardiñas y Balado, 2018, p. 132).

\section{Desafíos e importancia del desempeño docente}

La sociedad experimenta cambios vertiginosos en tiempos bastante cortos. La "sociedad contemporánea en la era de la información y de la incertidumbre" ha experimentado muchos cambios, exigiendo de esta manera los cambios de las políticas relacionadas con el desempeño docente. En este sentido, el desafío de las políticas educativas y del desempeño docente es preparar para enfrentar los cambios, la incertidumbre y la desigualdad enquistados en la sociedad actual (Pérez, 2010; en González y Subaldo, 2015). Por otro lado, se pretende "construir la sociedad del conocimiento partiendo de la sociedad de aprendizaje, con la escuela como principal actor" (Cuenca \& O’Hara, 2006; en González y Subaldo, 2015, 92). El estudiante y los docentes serán preparados para enfrentar una escuela y una sociedad muy diferentes a las de sus padres, en roles y funciones muy diferentes (Esteve, 2006; Mañú y Goyarrola, 2011; Ferrón, 2011; en González y Subaldo, 2015).

Entre las repercusiones del desempeño docente se suscriben: la satisfacción del docente, el desarrollo y la realización personal y profesional de los docentes, permitiendo de esta manera la presencia de "la calidad de la enseñanza y los aprendizajes de los alumnos"; el bienestar, el compromiso y la felicidad de la comunidad escolar (González y Subaldo, 2015). No se debe perder de vista que "una buena docencia implica también el dominio de las habilidades comunicativas para presentar el contenido de una manera organizada, coherente y dinámica" (Carlos, 2016, p. 298).

En este contexto, "la labor de los educadores ocupa en el país un lugar fundamental en la búsqueda de una educación de calidad, situada y pertinente" (Dimaté, Tapiero, González, Rodríguez y Arcila, 2017, p. 85).

En este sentido, si el sistema de formación docente lo constituye la formación inicial, la formación continua y la formación posgradual, el subsistema de evaluación del desempeño docente contendría las pruebas Saber Pro, pruebas de ingreso, pruebas de ascenso y pruebas de desempeño (Dimaté et al., 2017, p. 92).

Mejora "la calidad de los procesos educativos" (Martínez, 2016, en Tobón, Guzmán y Tobón, 2018). Se ha precisado la búsqueda del desempeño docente con enfoque de 
competencias, que describe "el comportamiento del profesor durante la conducción del proceso formativo", cuyo carácter es ético-humanista, desde la detección de los problemas, los requerimientos de la población, "la aplicación de los métodos científicos de la profesión y la integración docente-asistencial-investigativa" (Ortiz et al., 2018). La evaluación permite trabajar las fortalezas y mejorar diversos aspectos del desempeño docente (Quiriz \& Tobón, 2019).

\section{METODOLOGÍA}

En la presente investigación se ha abordado dos variables: el liderazgo pedagógico y el desempeño docente. La variable: liderazgo pedagógico ha sido atendida en sus dos dimensiones : liderazgo participativo y liderazgo consultivo. Por su parte, la variable: desempeño docente, en sus dimensiones: desempeño académico, desempeño administrativo, desempeño de interrelación y desempeño creativo.

El estudio ha recurrido a tres tipos de investigación: descriptivo, correlacional y transversal. La presencia del primero se explica por las descripciones (matemática y estadística) de algunas variables, específicamente las variables demográficas. El segundo tipo: correlacional recibe tal denominación, porque en la investigación se han realizado las correlaciones de las variables y sus respectivas dimensiones, específicamente durante el proceso de las respectivas pruebas de las hipótesis. Por su parte, el tipo de investigación transversal, denominado también transeccional, consiste en hacer un corte temporal durante el proceso de la investigación, con el propósito de aplicar el instrumento a los sujetos participantes $\mathrm{y}$, además, recabar la información y los datos requeridos y expuestos para los análisis pertinentes, de acuerdo con Hernández, Fernández y Baptista (2014).

\section{Diseño del estudio}

Para los efectos de los análisis estadísticos, se han diseñado cinco modelos estadísticos, con el propósito de probar las relaciones establecidas:

Modelo 1

$$
\mathrm{X}
$$

Modelo 2

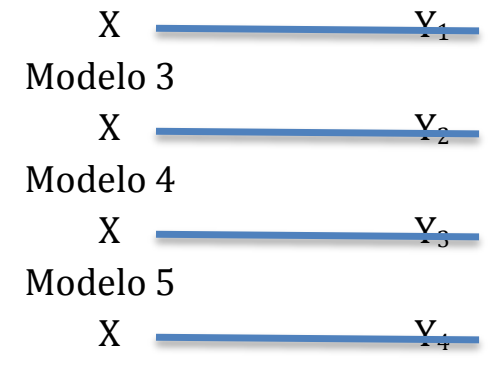

Donde:

$\mathrm{X}$ : Liderazgo pedagógico; $\mathrm{X}_{1}$ : Liderazgo participativo; $\mathrm{X}_{2}$ : Liderazgo consultivo; $\mathrm{Y}$ : Desempeño docente; $Y_{1:}$ Desempeño docente académico; $Y_{2:}$ Desempeño docente administrativo; $Y_{3}$ : Desempeño docente de interrelación; $Y_{4}$ : Desempeño docente creativo 


\section{La población y muestra del estudio}

La población total del personal docente en la Asociación Educativa Adventista Nor Oriental está constituida por 95 maestros, adscritos en los tres niveles: inicial, primerio y secundario, cuya descripción se presenta en el siguiente cuadro 1.

Cuadro 1. Distribución la población

\begin{tabular}{lcccccc}
\hline Institución educativa & Inicial & Primario & Secundario & Hombres & Mujeres \\
\hline Alto- Mayo de Rioja & 4 & 6 & 10 & 9 & 11 & 20 \\
Moyobamba & 4 & 7 & 11 & 10 & 12 & 22 \\
Nueva Cajamarca & 4 & 7 & 12 & 11 & 12 & 23 \\
San Martín de Tarapoto & 6 & 9 & 15 & 13 & 17 & 30 \\
\hline Totales & $\mathbf{1 8}$ & $\mathbf{2 9}$ & $\mathbf{4 8}$ & $\mathbf{3 3}$ & $\mathbf{4 2}$ & $\mathbf{9 5}$ \\
\hline
\end{tabular}

De los 95 maestros, que es la población, se tomó solamente 75, por libre participación y por la asistencia en el momento cuando fueron convocados. Es decir, solo asistieron 75 maestros, quienes conforman el número de participantes en el estudio, según los detalles registrados a continuación en el cuadro 2 :

Cuadro 2. Muestra especifica

\begin{tabular}{lcccccc}
\hline \multirow{2}{*}{ Institución educativa } & \multicolumn{3}{c}{ Nivel } & \multicolumn{3}{c}{ Género } \\
Inicial & Primario & Secundario & Hombres & Mujeres & \\
\hline Alto- Mayo de Rioja & 3 & 6 & 8 & 7 & 10 & 17 \\
Moyobamba & 3 & 6 & 9 & 6 & 12 & 18 \\
Nueva Cajamarca & 3 & 6 & 10 & 11 & 8 & 19 \\
San Martín de Tarapoto & 3 & 6 & 12 & 9 & 12 & 21 \\
\hline Totales & $\mathbf{1 2}$ & $\mathbf{2 4}$ & $\mathbf{3 9}$ & $\mathbf{3 3}$ & $\mathbf{4 2}$ & $\mathbf{7 5}$ \\
\hline
\end{tabular}

Técnicas de la recolección de datos

Galindo (2017) trabajó su investigación sobre el liderazgo directivo y el desempeño docente, en las IEI $\mathrm{N}^{\circ} 090$ y 091, ambas pertenecen al distrito de Jesús María, en el departamento de Lima, adscritas en la UGEL 03. Trabajó dos instrumentos, uno para cada variable. Para el presente estudio sirvió de base el "Cuestionario de Liderazgo Directivo", el cual presenta 21 ítems, agrupadas en dos dimensiones: "gestión de las condiciones para la mejora de los aprendizajes" y "orientación de los procesos pedagógicos para la mejora de los aprendizajes"; en la primera se consideraron 14 ítems y la segunda, 7.

Luego de la lectura, el análisis y la evaluación del instrumento referido, en el investigador y el guía, quedaron muchas dudas e imprecisiones, a pesar de que el documento tiene su fuente en el Ministerio de Educación. Las imprecisiones se observaron en el nombre de las dimensiones, el cual presenta un 
lenguaje complicado, impreciso, extenso, inadecuado, confuso y limitante.

Sobre la base del instrumento mencionado, se procedió a reorganizar los ítems, agrupándolos en sus respectivas variables. Se ha utilizado la técnica denominada encuesta, con el propósito de recabar los datos requeridos y sobre las dos variables: liderazgo pedagógico y desempeño docente. En este sentido, se ha trabajado dos instrumentos. Uno para recabar la información (los datos) sobre el liderazgo pedagógico y otro con el propósito de obtener la información (los datos) sobre el desempeño docente. En ambos instrumentos se ha trabajado la información demográfica, que comprende las variables: sexos, edad, estado civil, religión profesada, título profesional, grado académico, lugar de procedencia, nivel de enseñanza.

Por otro lado, el instrumento para trabajar el "Liderazgo pedagógico del director", permitió organizar los 15 ítems en dos variables: liderazgo participativo y liderazgo consultivo, 9 (1, 2, 3, 4, 5, 6, 7, 8 y 9) para el primero y $6(10,11,12,13,14$ y 15$)$ para el segundo. En el instrumento también se trabajó una escala tipo Likert: Nunca (1), a veces (2) y siempre (3), por su lenguaje familiar $y$ asequible para los participantes, facilitando el llenado del instrumento. El segundo instrumento para medir el desempeño docente ofrece 21 ítems, presenta cuatro dimensiones: desempeño docente académico, desempeño docente administrativo, desempeño docente de interrelación y desempeño académico creativo, con su respectivo número de ítems: cinco $(1,2,3,4$ y 5$)$, cuatro $(6,7,8$ y 9), ocho $(10,11,12,13,14,15,16,17)$ y cuatro $(18,19$, 20 у 21).

\section{Plan de análisis de datos}

Para los efectos de los análisis de los datos obtenidos durante el proceso de la investigación, se ha recurrido al uso de la estadística descriptiva y la estadística analítica. Mediante la estadística descriptiva se ha realizado la descripción de las variables cualitativas y cuantitativas, cuyos datos son presentados en tablas. La estadística analítica permite realizar la comprobación de las hipótesis, la normalidad de las variables.

\section{Validez y confiabilidad del instrumento}

Para la validez y la confiabilidad, se recurrió a los estudios teóricos, con el propósito de poseer el sustento teórico respectivo; además sobre la existencia de instrumentos ya validados se procedió a estudiar la pertinencia de los mismos, con la opinión de asesores y expertos sobre las mismas variables. En este sentido, se procedió a realizar la validación y la confiabilidad de los instrumentos. La validación estadística se presenta a continuación en la tabla 1 :

Tabla 1. Análisis de confiabilidad

Estadísticas de fiabilidad

Alfa de Cronbach

, 940
$\mathrm{N}$ de elementos

36 
Luego de realizar el correspondiente análisis de confiabilidad de Alfa de Cronbach, se determinó un $94 \%$ de confiabilidad, permitiendo afirmar que este instrumento (cuestionario) es altamente confiable.

\section{Aspectos éticos}

Para los efectos de la investigación, se realizaron las gestiones ante las autoridades de la Universidad Adventista de Chile (UNACH) y las autoridades eclesiásticas y educativas de la Asociación Educativa Nor Oriental (ASEANOR); además, se conversó con los docentes de la misma asociación, explicando el propósito de la participación, dejando constancia, asimismo, que la información recabada es totalmente anónima, sin generar perjuicio alguno a los participantes. Finalmente, se procedió a firmar el documento denominado Consentimiento informado.

RESULTADOS

\section{Prueba de correlación de liderazgo pedagógico y desempeño docente académico}

Tabla 2. Prueba de correlación Rho de Spearman entre el liderazgo pedagógico y el desempeño docente académico

\begin{tabular}{lccc}
\hline & \multicolumn{3}{c}{ Liderazgo pedagógico } \\
& $\begin{array}{c}\text { Rho } \\
\text { Spearman }\end{array}$ & $\begin{array}{c}\text { Sig. } \\
\text { (bilateral) }\end{array}$ & $\mathbf{N}$ \\
\hline Desempeño docente académico &, $427^{* *}$ & .000 & 75 \\
\hline
\end{tabular}

Mediante el análisis estadístico de correlación de Rho Spearman se obtuvo el resultado de 0,427 y un p valor igual a 0.000 (p valor $<0,05$ ); como se muestra en la tabla 2 , por lo tanto, se rechaza la hipótesis nula y se acepta la hipótesis alterna; el liderazgo pedagógico se relaciona significativamente con el desempeño docente académico, se observa que la relación entre las variables estudiadas es directa; es decir, a mejor liderazgo pedagógico, mejor será el desempeño docente académico.

\section{Prueba de correlación de liderazgo pedagógico y desempeño docente administrativo}

Tabla 3. Prueba de correlación Rho de Spearman entre el liderazgo pedagógico y el desempeño docente administrativo

\section{Liderazgo pedagógico}

Rho Spearman Sig. (bilateral) N

\begin{tabular}{lll}
\hline Desempeño docente administrativo &, $440^{* *}$ & .000 \\
\hline
\end{tabular}


A través del análisis estadístico de correlación de Rho Spearman presentado en la tabla 3 se obtuvo el resultado de 0,440 y un p valor igual a 0.000 ( $p$ valor $<0,05$ ); por lo tanto, se rechaza la hipótesis nula y se acepta la hipótesis alterna; es decir, el liderazgo pedagógico se relaciona significativamente con el desempeño docente administrativo. La relación entre las variables estudiadas es directa; vale decir, a mejor liderazgo pedagógico, mejor será el desempeño docente administrativo.

\section{Prueba de correlación de liderazgo pedagógico y desempeño docente de interrelación}

Tabla 4. Prueba de correlación Rho de Spearman entre el liderazgo pedagógico y el desempeño docente de interrelación

\begin{tabular}{|c|c|c|c|}
\hline & \multicolumn{3}{|c|}{ Liderazgo pedagógico } \\
\hline & Rho Spearman & Sig. (bilateral) & $\mathbf{N}$ \\
\hline Desempeño docente de interrelación & ,388** & .001 & 75 \\
\hline
\end{tabular}

A través del análisis estadístico de correlación de Rho Spearman que se mostró en la tabla 4 se obtuvo el resultado de 0,338 y un p valor igual a 0.001 ( $\mathrm{p}$ valor $<0,05$ ); por lo tanto, se rechaza la hipótesis nula y se acepta la hipótesis alterna; o sea, el liderazgo pedagógico se relaciona significativamente con el desempeño docente de interrelación. La relación entre las variables estudiadas es directa; es decir, a mejor liderazgo pedagógico, mejor será el desempeño docente de interrelación.

\section{Prueba de correlación de liderazgo pedagógico y desempeño docente creativo}

Tabla 5. Prueba de correlación Rho de Spearman entre el liderazgo pedagógico y el desempeño docente creativo

\begin{tabular}{lccc}
\hline & \multicolumn{3}{c}{ Liderazgo pedagógico } \\
& Rho Spearman & Sig. (bilateral) & N \\
\hline Desempeño docente creativo &, $602^{* *}$ & .000 & 75 \\
\hline
\end{tabular}

En la tabla 5 se mostró el análisis estadístico de correlación de Rho Spearman, en donde se obtuvo el resultado de 0,602 y un p valor igual a 0.000 ( $\mathrm{p}$ valor $<0,05$ ); se rechaza la hipótesis nula y se acepta la hipótesis alterna; es decir, el liderazgo pedagógico se relaciona significativamente con el desempeño docente creativo. La relación entre las variables estudiadas es directa; en otros términos, a mejor liderazgo pedagógico, mejor será el desempeño docente creativo. 


\section{Prueba de correlación de liderazgo pedagógico y desempeño docente}

Tabla 6. Prueba de correlación Rho de Spearman entre el liderazgo pedagógico y el desempeño docente

\begin{tabular}{lccc}
\hline & & Liderazgo pedagógico & \\
& Rho Spearman & Sig. (bilateral) & N \\
\hline Desempeño docente &, $543^{* *}$ & .000 & 75 \\
\hline
\end{tabular}

A través del análisis estadístico de correlación de Rho Spearman, se obtuvo el resultado de 0,543 y un $\mathrm{p}$ valor igual a $0.000(\mathrm{p}$ valor < 0,05); por lo tanto, se rechaza la hipótesis nula y se acepta la hipótesis alterna; es decir, el liderazgo pedagógico se relaciona significativamente con el desempeño docente. Además, la relación entre las variables estudiadas es directa; vale decir que, a mejor liderazgo pedagógico, mejor será el desempeño docente según se destacó en la tabla 6 .

\section{Discusión}

En esta sección de la investigación, se pretende construir la discusión en torno de las hipótesis; es decir, en relación con los cuatro modelos: tres específicos y con el general. Sin embargo, se reconoce que las investigaciones consultadas no han abordado las mismas variables y dimensiones; en este sentido, la presente investigación se realizó siguiendo algunos lineamientos establecidos por el Ministerio de Educación del Perú, cuyas condiciones, al final de cuentas, generaron una limitación enorme para el desarrollo de la investigación, razón por la cual las comparaciones son totalmente parciales, excepto la primera variable: liderazgo pedagógico.

Por su parte, Jiménez (2017) realiza su estudio sobre liderazgo pedagógico y trabajo en equipo de los docentes en el nivel inicial, con el propósito de responder a la cuestión: “¿Cuál es la relación entre el liderazgo pedagógico y el trabajo en equipo en los docentes de educación inicial de la zona urbana Huaral, 2016?", habiendo trabajado tres dimensiones del liderazgo pedagógico: planificación, organización y evaluación, en el marco de la hipótesis suya: "Existe relación entre el liderazgo pedagógico y el trabajo en equipo en los docentes de educación inicial de la zona urbana de Huaral, 2016", lo que significa haber realizado un investigación de enfoque cuantitativo, con un diseño transversal, descriptivo, en 7 instituciones educativas, en una muestra de 67 docentes, a quienes se les aplicó dos instrumentos con el propósito de recabar los datos requeridos.

Entre sus resultados, se halló el p-valor = 0,001 , el cual es inferior al nivel de significancia $(\alpha=0,05)$; por esta razón se rechazó la hipótesis nula y se aceptó la hipótesis de investigación, llegando a la conclusión de sí "existe relación entre el liderazgo pedagógico y el trabajo en equipo en los docentes de educación inicial de la zona urbana de Huaral", cuyo valor de la Rho de Spearman es igual a 0,803; es decir, "ambas variables están relacionadas significativamente"; además "presentan una relación directa y de nivel alto"; por lo tanto, "a mayores valores de liderazgo pedagógico, existirán mayores niveles de trabajo en equipo". 
Este estudio tiene una relación más cercana, más próxima a la hipótesis específica 1 del presente estudio; cuyo texto dice: El liderazgo pedagógico se relaciona significativamente con el desempeño docente académico, en la Asociación Educativa Adventista Nor Oriental. En este sentido, Jiménez (2017) investigó "el trabajo en equipo en los docentes"; en cambio, la investigación, objeto de exposición, abordó el desempeño docente; en la cual se registran los respectivos resultados: existe relación del liderazgo pedagógico con desempeño docente académico, cuyo análisis estadístico de correlación de Rho Spearman revela el resultado de 0,427 y un $\mathrm{p}$ valor igual a 0.000 ( $\mathrm{p}$ valor $<0,05$ ); es decir, se observa que la relación entre las variables estudiadas es directa; vale decir que a mejor liderazgo pedagógico, mejor será el desempeño docente académico.

Saavedra (2018) realiza la investigación con mucha aproximación a la presente. Trabaja el liderazgo pedagógico directoral y los procesos de los aprendizajes, entendiendo que esta última variable se aproxima a la variable: desempeño docente académico. El estudio fue realizado en docentes (23) y en padres de familia (07), a diferencia de la presente, se trabajó solamente con los docentes. El diseño "corresponde al tipo de investigación es expost-facto de grupo criterio", el presente no. El estudio "concluye que el liderazgo pedagógico directoral influye de manera altamente significativa en los procesos de aprendizaje", "con un nivel de confianza del $99 \%$ y $1 \%$ de probabilidad de error".

La investigación de Huamán (2018) aborda el liderazgo pedagógico directoral y la gestión de los aprendizajes, en Andamarca, región de Ayacucho, una comunidad andina y quechua hablante, con el objetivo de determinar la relación de ambas variables, en correspondencia a su respectiva hipótesis: "El liderazgo pedagógico directoral se relaciona significativamente con la gestión de los aprendizajes de los estudiantes de la Institución Educativa Nro. 24073 Luis Alfaro Calle de Andamarca". Para lograr el objetivo, se realizó una consulta al personal docente (20) y a los padres de familia (10), mediante los instrumentos respectivos, en el marco de un enfoque cuantitativo, descriptivo, correlacional.

Entre sus resultados, se establece la relación entre las variables: liderazgo pedagógico directoral y gestión de los aprendizajes de los estudiantes, cuyo “coeficiente de correlación $r=0,8755$ ". En conclusión, "el liderazgo pedagógico directoral se relaciona significativamente con la gestión institucional de los aprendizajes de los estudiantes", "con un nivel de confianza del 99 $\%$ y $1 \%$ de probabilidad de error".

Por su parte, la presente investigación responde a la hipótesis: El liderazgo pedagógico se relaciona significativamente con el desempeño docente administrativo, en la Asociación Educativa Adventista Nor Oriental. Las variables: liderazgo pedagógico $\mathrm{y}$ desempeño docente administrativo presentan el estadístico de correlación de Rho Spearman de 0,440 y un p valor igual a 0.000 (p valor < $0,05)$, concluyéndose que se observa la relación entre las variables estudiadas es directa; es decir, a mejor liderazgo pedagógico, mejor será el desempeño docente administrativo.

Roldán (2014) investigó sobre "liderazgo pedagógico del directivo y la autoevaluación del desempeño docente en las instituciones educativas de la red 2 de la Ugel 03 , Cercado de Lima", en un contexto de estudio básico, estableciendo la relación de las variables, no experimental, descriptivo, transaccional, en una población de 190 docentes, mediante un muestro probabilístico, aleatorio simple, cuyos datos fueron recopilados mediante dos cuestionarios, uno para cada variable. Su 
hipótesis general fue: "El liderazgo pedagógico del directivo tiene relación positiva $\mathrm{y}$ significativa con la autoevaluación del desempeño docente en las instituciones educativas de la red 2 de la Ugel 03 distrito de Cercado de Lima, 2014". Según este estudio, las variables estudiadas tienen una "correlación positiva baja", cuyo Rho $=0,293$, rechazando la hipótesis nula y aceptando la hipótesis alternativa.

El estudio presenta la hipótesis general: El liderazgo pedagógico se relaciona significativamente con el desempeño docente. Es decir, solamente se trabajó el liderazgo pedagógico, a diferencia del estudio de Roldan Rosadio, quien estudió el liderazgo del directivo del director. Además, estudió la variable: autoevaluación del desempeño docente, a diferencia del presente que aborda: el desempeño docente, al interior del país.

En términos de los resultados propiamente dichos, el estudio antecedente presenta el Rho $=$ 0,293, el rechazo a la hipótesis nula y la aceptación a la hipótesis alternativa. Por parte de la investigación presente, las variables: liderazgo pedagógico y desempeño docente, presentan el análisis estadístico de correlación de Rho Spearman, cuyo resultado es 0,543 y un $\mathrm{p}$ valor igual a 0.000 ( $\mathrm{p}$ valor $<0,05$; se observa que la relación entre las variables estudiadas es directa; es decir, a mejor liderazgo pedagógico, mejor será el desempeño docente.

\section{CONCLUSIONES}

Se presenta a continuación cinco conclusiones: cuatro específicas que corresponden a las hipótesis específicas (modelos específicos) y una conclusión general (modelo general), la cual tiene relación con la hipótesis general de la investigación.

Estudiada la relación entre las variables: liderazgo pedagógico y desempeño docente académico, además dado el análisis estadístico de correlación de Rho Spearman y el resultado de 0,427 y el $p$ valor igual a 0.000 (p valor < $0,05)$, se rechaza la hipótesis nula y se acepta la hipótesis de investigación, porque el liderazgo pedagógico se relaciona significativamente con el desempeño docente académico. Se concluye que existe relación directa entre las variables estudiadas; a mejor liderazgo pedagógico, mejor será el desempeño docente académico.

La relación es directa entre las variables: liderazgo pedagógico y desempeño docente administrativo, cuyo sustento es el análisis estadístico de correlación de Rho Spearman, el resultado de 0,440 y el $p$ valor igual a $0.000(p$ valor < 0,05); en este sentido, se rechaza la hipótesis nula y se acepta la hipótesis de investigación; es decir, el liderazgo pedagógico se relaciona significativamente con el desempeño docente administrativo; en efecto, se concluye que existe una relación directa entre las variables referidas; vale decir que a mejor liderazgo pedagógico, mejor será el desempeño docente administrativo.

El liderazgo pedagógico se relaciona significativamente con el desempeño docente de interrelación; las variables: liderazgo pedagógico y desempeño docente de interrelación, mantienen una relación significativa, dejando a la vista el análisis estadístico de correlación de Rho Spearman, cuyo resultado es 0,338 con un $p$ valor igual a 0.001 ( $\mathrm{p}$ valor $<0,05$ ); por lo tanto, se rechaza la hipótesis nula y se acepta la hipótesis investigación; en conclusión, las variables mencionadas guardan una relación directa; en este contexto, se sostiene que a mejor liderazgo pedagógico, mejor será el desempeño docente de interrelación.

El liderazgo pedagógico se relaciona significativamente con el desempeño docente creativo, Al respecto, se observa la relación entre las variables: liderazgo pedagógico y desempeño docente creativo, de acuerdo con el 
análisis estadístico de correlación de Rho Spearman, el resultado de 0,602 y un p valor igual a 0.000 (p valor $<0,05$ ); se rechaza la hipótesis nula y se acepta la hipótesis de investigación; en conclusión, el liderazgo pedagógico se relaciona significativamente con el desempeño docente creativo; es decir, la relación entre las variables estudiadas es directa; en otras palabras, se puede decir que a mejor liderazgo pedagógico, mejor será el desempeño docente creativo.

El liderazgo pedagógico se relaciona significativamente con el desempeño docente. Se precisa la relación de las variables: liderazgo pedagógico y desempeño docente, cuyo análisis estadístico de correlación de Rho Spearman presenta el resultado de 0,543 y un p valor igual a 0.000 ( $p$ valor $<0,05$ ); se rechaza la hipótesis nula y se acepta la hipótesis de investigación; en conclusión, el liderazgo pedagógico se relaciona significativamente con el desempeño docente; dicho de otro modo, la relación entre las variables estudiadas es directa; es decir, a mejor liderazgo pedagógico, mejor será el desempeño docente.

\section{REFERENCIAS}

Carlos, J. (2016). ¿Qué y cómo evaluar el desempeño docente? Una propuesta basada en los factores que favorecen el aprendizaje. Propósitos Y Representaciones, 4(2), 285321.

https://doi.org/http://dx.doi.org/10.20511 /pyr2016.v4n2.124

Cortez, J. A. (2018). Desarrollo de competencias en el proceso de enseñanza y aprendizaje del área de comunicación Plan de acción. [Tesis, Pontificia Universidad Católica del Perú]. Retrieved from http://tesis.pucp.edu.pe/repositorio/handl e/20.500.12404/10644

Dimaté, C., Tapiero, O., González, C. I., Rodríguez, R., \& Arcila, M. A. (2017). La evaluación del desempeño docente. Folios, (46), 83-95. Retrieved

from http://www.redalyc.org/articulo.oa?id=461 42596007

Estrada, E., \& Mamani, H. (2020). Compromiso organizaional y desempeño docente en las instituciones de educación básica. Rev. Innova Educ, 2(1), 133-146. https://doi.org/https://doi.org/10.35622/j .rie.2020.01.008

Fernández, E. M. (2018). Aplicación de los procesos pedagógicos y didácticos en el desarrollo de las sesiones de aprendizaje de comprensión lectora. [Tesis, Pontificia Universidad Católica del Perú]. Retrieved from

http://tesis.pucp.edu.pe/repositorio/handl e/20.500.12404/11428

Fernández, R., \& Martín, M. (Eds.). (2014). La formación del profesorado en Iberoamérica: proyecciones en la práctica. Alcalá de Henares, España: Fundación Santillana. Retrieved from https://www.researchgate.net/publication /333508077_LA_FORMACION_DEL_PROFE SORADO_EN_IBEROAMERICA_PROYECCIO NES_EN_LA_PRACTICA

Flores, F., Gatica, F., Sánchez, M., \& Martínez, A. (2017). Evolución de la evaluación del desempeño docente en la Facultad de Medicina; evidencia de validez y confiabilidad. Investigación En Educación Médica, 6(22), 96-103. https://doi.org/10.1016/j.riem.2016.06.00 4

Flores, F., Sánchez, M., \& Martínez, A. (2016). Modelo de Predicción del Rendimiento Académico de los Estudiantes del Ciclo Básico de la Carrera de Medicina a partir de la Evaluación del Desempeño Docente. Revista Mexicana de Investigación Educativa, 21(70), 975-991. Retrieved from http://www.scielo.org.mx/scielo.php?pid=S 140566662016000300975\&script=sci_arttext

Galindo Ynga, D. M. (2017). Liderazgo directivo y desempeño docente en las IEI $N^{\circ} 090$ y 091 del distrito de Jesús María - UGEL 03 , 2017. [Tesis de maestría, Universidad César Vallejo]. $\quad$ Retrieved from 
https://repositorio.ucv.edu.pe/handle/20.5 $00.12692 / 14180$

Gento, S., González, R., \& Silfa, H. O. (2020). Dimensión afectiva del liderazgo pedagógico del docente. Revista Complutense de Educación, 31(4), 485495.

https://doi.org/https://doi.org/10.5209/ rced.65635

González, J., \& Subaldo, L. (2015). Opiniones sobre el desempeño docente y sus repercusiones en la satisfacción profesional y personal de los profesores. Educación, XXIV(47), 90-114. Retrieved from

http://revistas.pucp.edu.pe/index.php/ed ucacion/article/view/14247/14865

Hernández, R., Fernández, C., \& Baptista, P. (2014). Metodología de la investigación (Sexta aedi). México D.F: McGraw Hill Interamericana Editores, S.A. de C.V. https://doi.org/10.1017/CB0978110741 5324.004

Huamán Condorcule, M. (2018). El liderazgo pedagógico directoral y la gestión de los aprendizajes de los estudiantes de la Institución Educativa Nro. 24073 Luis Alfaro Calle de Andamarca - Lucanas, región Ayacucho. [Tesis de grado académico de maestro, Universidad Nacional de Educación Enrique Guzmán y Valle]. Retrieved from https://repositorio.une.edu.pe/handle/U NE/1826

Hurtado, L. C. (2017). El liderazgo y el desempeño gerencial de los directores, según percepción de los docentes en las instituciones educativas de Fe y Alegría en Comas, 2016. [Tesis de grado académico de maestro, Universidad Nacional de Educación Enrique Guzmán y Valle]. Retrieved from https://repositorio.une.edu.pe/bitstream /handle/UNE/1317/TM AD-Gp 3273 H1 Hurtado

Solis.pdf?sequence $=1$ \&isAllowed $=y$

Jiménez, A. (2017). Liderazgo pedagógico y trabajo en equipo en los docentes de educación inicial de la zona urbana de de Huaral-2016. [Tesis de magíster, Universidad César Vallejo]. Retrieved from https://repositorio.ucv.edu.pe/handle/20 $.500 .12692 / 8374$

Llorent, V., Cobano, V., \& Navarro, M. (2017). Liderazgo pedagógico y dirección escolar en contextos desfavorecidos. Revista Española de Pedagogía, 75(268), 541-564. https://doi.org/10.22550/REP75-3-201704

Martínez, J. E., Castillo, L. C., \& Granda, V. D. (2017). Formación inicial del docente de educación física y su desempeño profesional. EmásF, Revista Digital de Educación Física, 8(48), 83-95. Retrieved from https://dialnet.unirioja.es/servlet/articul o? codigo $=6121667$

Mellado, M. E., Chaucono, J. C., \& Villagra, C. P. (2017). Creencias de directivos escolares: implicancias en el liderazgo pedagógico. Psicologia Escolar E Educacional, 21(3), 541-548. https://doi.org/10.1590/21753539/2017/0213111102

Ortiz, M., Borges, L., Rodríguez, I., Sardiñas, M. E., \& Balado, R. (2018). Modelo pedagógico para el mejoramiento del desempeño docente con enfoque de competencias en la especialidad de pediatría. Revista Habanera de Ciencias Médicas, 17(1), 129-143. Retrieved from http://www.revhabanera.sld.cu/index.ph p/rhab/article/view/2106

Penichel, R., Ramón, C., Guzmán, C., \& Mora, N. (2020). Factores que afectan el desempeño docente en centros de alta y baja eficacia en México. REICE. Revista Iberoamericana Sobre Calidad, Eficacia $Y$ Cambio En Educación, 18(2), 77-95. https://doi.org/https://revistas.uam.es/i ndex.php/reice/article/view/reice2020.1 8.2.004

Pimienta, J. H. (2014). Elaboración y validación de un instrumento para la medición del desempeño docente basado en competencias. REDU Revista 
de Docencia Universitaria, 12(2), 231$250 . \quad$ Retrieved from https://polipapers.upv.es/index.php/RE DU/article/view/5648

Quiriz, T., \& Tobón, S. (2019). Fortalezas y aspectos a mejorar en la evaluación del desempeño docente en México considerando la socioformación y experiencias internacionales. RA XIMHAI, 15(1), 101-115. https://doi.org/doi.org/10.35197/rx.15.01. 2019.08.tq

Quiroga, M., \& Aravena, F. (2017). Jefes de UTP nóveles en Chile: desafíos del liderazgo pedagógico. Perspectiva Educacional, 56(3), 76-97. https://doi.org/10.4151/07189729Vol.56-Iss.3-Art.506

Quispe, M. (2020). La gestión pedagógica en la mejora del desempeño docente. Investigación Valdizana, 14(1), 7-14. Retrieved from https://dialnet.unirioja.es/servlet/articulo? codigo $=7409389$

Ramos, L., \& Rueda, M. (2020). Rasgos distintivos de las evaluaciones formativas para el desempeño docente. Perfiles Educativos, XLII(169), 144-159. https://doi.org/https://doi.org/10.22201/i isue.24486167e.2020.169.59287

Roca, P. M., \& Alonso, L. A. (2020). Propuesta de la competencia de liderazgo pedagógico a desarrollar en los maestros de Educación Básica. Luz, XIX(1), 109-123. Retrieved from https://luz.uho.edu.cu/index.php/luz/articl e/view/1020

Roldán, M. A. (2014). Liderazgo pedagógico del directivo y la autoevaluación del desempeño docente, Lima 2014. [Tesis de magíster, Universidad César Vallejo]. Retrieved from https://repositorio.ucv.edu.pe/handle/20.5 $00.12692 / 4970$

Romero, C. (2016). Liderazgo Directivo para la Mejora Escolar: Regulación, Formación y Prácticas. Un Estudio sobre Directores Escolares en Argentina. Avances En Liderazgo Y Mejora de La Educación, 474478. Retrieved from https://repositorio.uam.es/bitstream/hand le/10486/679653/109_liderazgo_romero_C ILME_2017.pdf?sequence=1\&isAllowed $=\mathrm{y}$

Saavedra, L. (2018). El liderazgo pedagógico directoral y los procesos de aprendizaje en la Institución Educativa Nro. 2023 Augusto Salazar Bondy de San Martín de Porres Lima. [Tesis de grado académico de maestro, Universidad Nacional de Educación Enrique Guzmán y Valle]. Retrieved from https://repositorio.une.edu.pe/handle/UNE $/ 1912$

Tobón, S., Guzmán, C. E., \& Tobón, B. (2018). Evaluación del Desempeño Docente en México: Del Proyecto de Enseñanza al Proyecto Formativo Evaluation of Teaching Performance in Mexico: From the Teaching Project to the Formative Project. Atenas Revista Científico Pedagógica, 1(41), 18-33. Retrieved from https://www.redalyc.org/jatsRepo/4780/4 78055151002/478055151002.pdf

Vela, G. A., Cáceres, T. J., Vela, A. F., \& Gamero, H. E. (2020). Liderazgo pedagógico en Arequipa-Perú: Competencias directivas. Revista de Ciencias Sociales (Ve), XXVI(Número especial 2), 376-400. Retrieved from https://dialnet.unirioja.es/servlet/articulo? codigo $=7599952$

Vela Quico, G. A. (2017). Teorías implícitas de los directores para el liderazgo pedagógico en los Ceba de Arequipa (estudio de casos). [Tesis de maestría, Universidad Nacional de San Agustín de Arequipa]. Retrieved from http://repositorio.unsa.edu.pe/handle/UNS A/4368

Villegas, C. A. (2018). Mejoramiento de la práctica pedagógica en matemática. [Tesis, Pontificia Universidad Católica del Perú]. Retrieved from http://tesis.pucp.edu.pe/repositorio/handl e/20.500.12404/10402 